\title{
Acute disseminated encephalomyelitis, with mass
necrosis of the spinal cord, probably due to antitetanus serum
}

\author{
A. A. Miller AND F. RAMSDEN \\ From the Group Laboratory, Preston Royal Infirmary
}

SYNOPSIS The clinical and pathological findings are described of a fatal case of acute haemorrhagie leucoencephalitis and disseminated encephalomyelitis with acute necrosis of the white matter $\vec{\omega} f$ the spinal cord. It is suggested that the reaction was a severe immunological response of an allergive nature, probably due to antitetanus serum.

The case described in this paper is of great interest for the following reasons: Acute disseminated encephalomyelitis and acute haemorrhagic leucoencephalitis with massive necrosis of the white matter of the spinal cord is an extremely rare condition which we have not found described in man in the available literature. Demyelinating encephalomyelitis after the administration of prophylactic serum has been reported only infrequently. In this case the onset of the neurological symptoms was not heralded by any local upset or skin eruption.

\section{CASE REPORT}

The patient, a healthy girl aged 23 years, was admitted to the North Lonsdale Hospital following a scooter accident: injuries were a simple fracture of the mid shaft of the left femur with abrasions of the left thigh and right elbow. Immediate treatment included the intramuscular injection of penicillin, subcutaneous injection of $1 \mathrm{ml}$. of antitetanus serum, and 1 grain of morphia.

Her condition remained satisfactory until the fifteenth day when she developed acute retention of urine followed, some hours later, by flaccid paraplegia, weakness of the left arm, unequal pupils, and sensory loss below the second thoracic segment.

On the seventeenth day she was transferred to the Neurosurgical Unit of Preston Royal Infirmary under the care of Mr. R. A. Daws. At the time of admission she was fully conscious, cooperative, and well orientated. There was flaccid paraplegia; the only reflex demonstrable in the legs was a depressed right knee jerk. Loss of sensation to pin prick reached to the level of the second thoracic segment and there was dulling of light touch sensation over this area. Upper limb reflexes were

Received for publication 15 November 1962. grossly exaggerated; the left arm was weak and incoordinated; the jaw jerk was brisk. Upward movement 0 the eyes was restricted and bilateral papilloedema wås present.

Radiological examination of the skull and sping column and a right carotid angiogram showed no abnormality; the transverse fracture of the mid shaft of the left femur was confirmed radiologically.

Cerebrospinal fluid was under normal pressure; cellul and chemical constituents were normal. Serologicat tests, including the Wassermann reaction and P.P.R were negative. Haematological investigations did nof show any evidence of anaemia: white cells were increased (23,000 per c.mm.) due to polymorphonuclear leucocytosis; the erythrocyte sedimentation rate was slight raised; serum electrolytes were normal apart from : slightly low chloride level (91.6 mEq./1.); blood urea was $185 \mathrm{mg}$. per $100 \mathrm{ml}$.

The urine contained numerous pus cells and gave profuse growth of a coliform organism when cultured.

The patient was examined by Dr. N. Gordon, the consultant neurologist, who diagnosed acute encephald myelitis, probably of allergic origin. Treatment with antihistamines was instituted and tetracyclines were given to control urinary infection.

Her condition deteriorated rapidly, extensive paralysis developed; the patient became drowsy and confused Lumbar puncture on the twenty-second day showed pressure within the normal range. Examination of the cerebrospinal fluid did not show abnormality. On the twenty-third day intravenous steroids were given. The patient died in coma early next morning. Death was of the twenty-fourth day after the administration of serum and nine days after the onset of neurological symptom?

During life she worked in a pharmaceutical laboratoros where most of her time was spent in washing up duti involving innocuous substances; more recently she had sampled penicillin and streptomycin. She had never been exposed to poisonous substances such as cyanides. 
NECROPSY

The examination was carried out about 48 hours after death, the body being refrigerated in the interim period. The deceased was a sparely built, well-nourished young woman. Abrasions on the left thigh and right elbow were noted; the fracture of the left femur was not united. Injuries to the scalp, skull, spine, and other bones were not found.

The lungs showed congestion of all lobes with bilateral, basal haemorrhagic bronchopneumonia. The heart was structurally normal: the left ventricular myocardium was flaccid, toneless and congested. Abdominal organs were congested. The spleen was enlarged, soft, congested, and contained a large amount of pulp. The bladder showed haemorrhagic cystitis and the kidneys the appearances of pyelonephritis. All the endocrine glands appeared normal.

The cause of death was respiratory failure, due to acute disseminated encephalomyelitis with maximal involvement of the spinal cord.

\section{PATHOLOGY OF THE CENTRAL NERVOUS SYSTEM}

MACROSCOPIC FINDINGS The brain weighed $1,185 \mathrm{~g}$. The leptomeninges were acutely congested; pinpoint haemorrhages were seen on both cerebral convexities but without exudate. The main cerebral arteries appeared normal; cerebral veins and dural sinuses contained fluid blood with small postmortem clots. On coronal section the ventricles were of normal size; the grey and white matter cf both cerebral hemispheres and, to a lesser extent the brain-stem, showed slight diffuse congestion and oedema. Numerous petechial haemorrhages were seen in the white matter of the posterior frontal and anterior parietal regions of both sides and in both corpora striata; diffuse haemorrhages were present in the right postero-lateral region of the pons and the white matter of the right cerebellum. Macroscopic lesions were not seen in the corpus callosum. Areas of softening were not found in the brain substance.

The spinal cord, from lower cervical to upper lumbar segments, was swollen and soft; the leptomeninges were intensely congested but there was no meningeal exudate (Fig. 1). Section of the dorsal segments after fixation showed soft grey necrotic surfaces: the cervical and lumbo-sacral parts were oedematous and congested but were firmer than the dorsal cord.

MATERIALS AND METHODS Samples were taken from every segment of the cord together with nerve roots and root ganglia; from the lower and upper medulla oblongata, the pons, cerebellum, brain-stem, basal ganglia, the

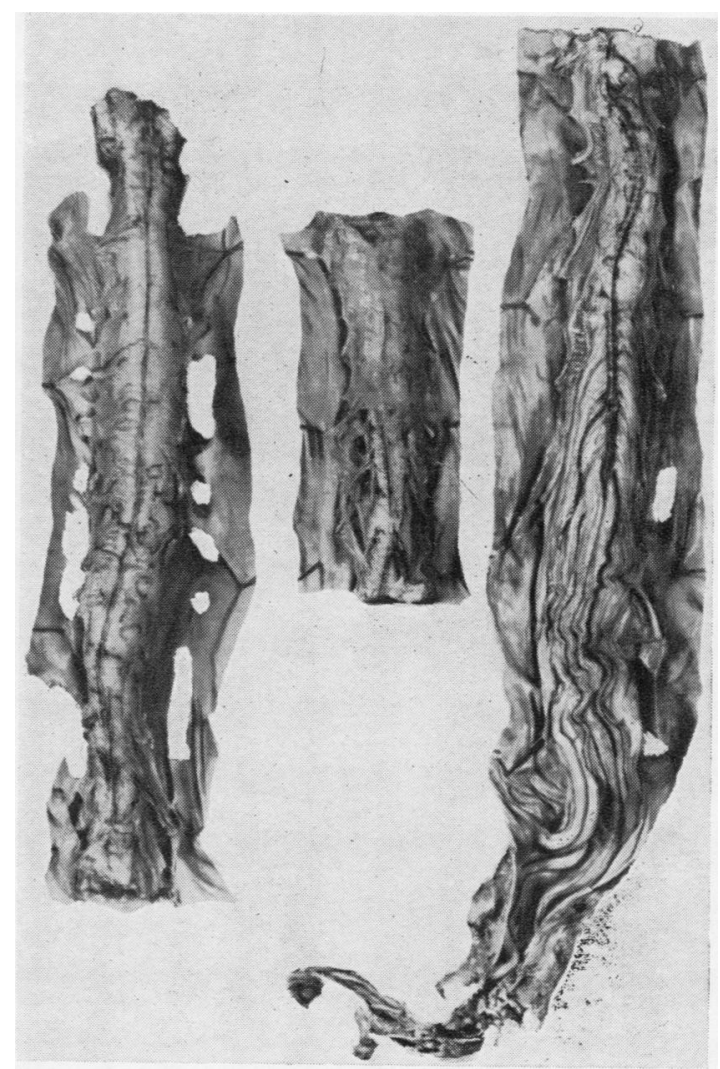

FIG. 1. Posterior aspect of spinal cord showing congestion of leptomeninges and oedematous swelling of the dorsal cord.

frontal, parietal and occipital cortex and white matter and the corpus callosum; and from the cranial nerves. Paraffin, celloidin, and freezing methods were used to prepare sections which were stained by a variety of techniques including haematoxylin and eosin, van Gieson's stain, and phosphotungstic acid haematoxylin. Loyez haematoxylin and Weigert-Pal preparations were used for demonstration of myelin. Frozen sections were treated by Weil Davenport's method and by Penfield's modification of Hortega's silver carbonate technique to demonstrate neuroglial cells and neurofibrils.

Sudan III and IV, sudan black B, and periodic acidSchiff methods were used to demonstrate myelin breakdown products.

MICROSCOPIC FINDINGS The leptomeninges appeared normal apart from congestion and extravasation of red blood cells. In the brain substance the main finding was numerous foci of perivascular demyelination and cellular cuffing around small veins; the cellular sleeve consisted of histiocytes and granular amoeboid microglial cells with irregularly lobed 


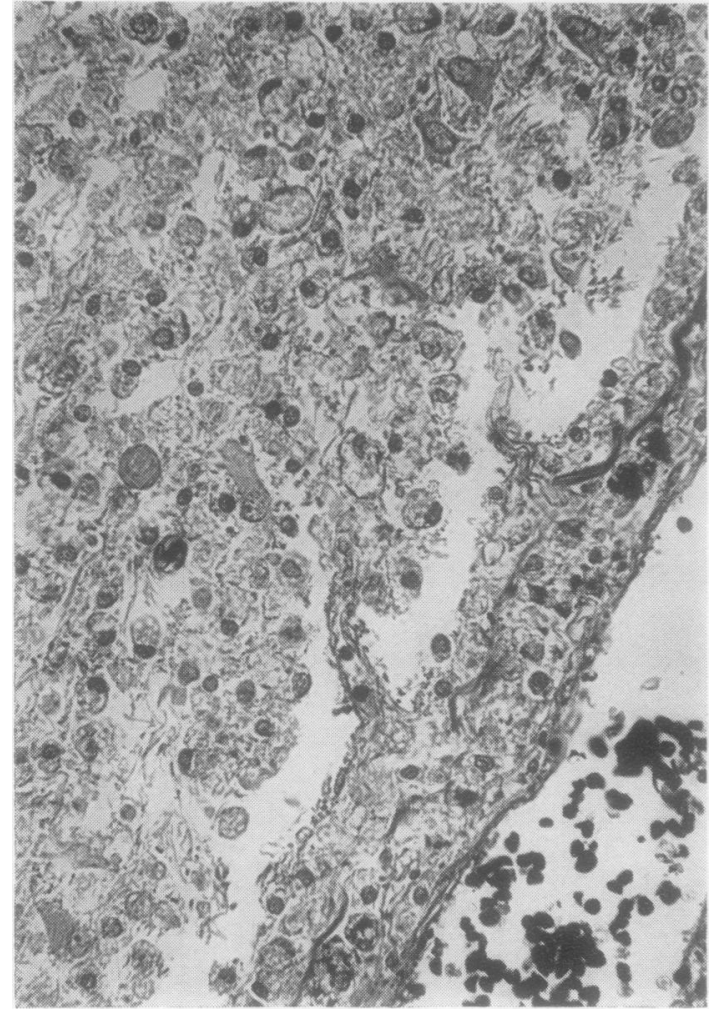

FIG. 2

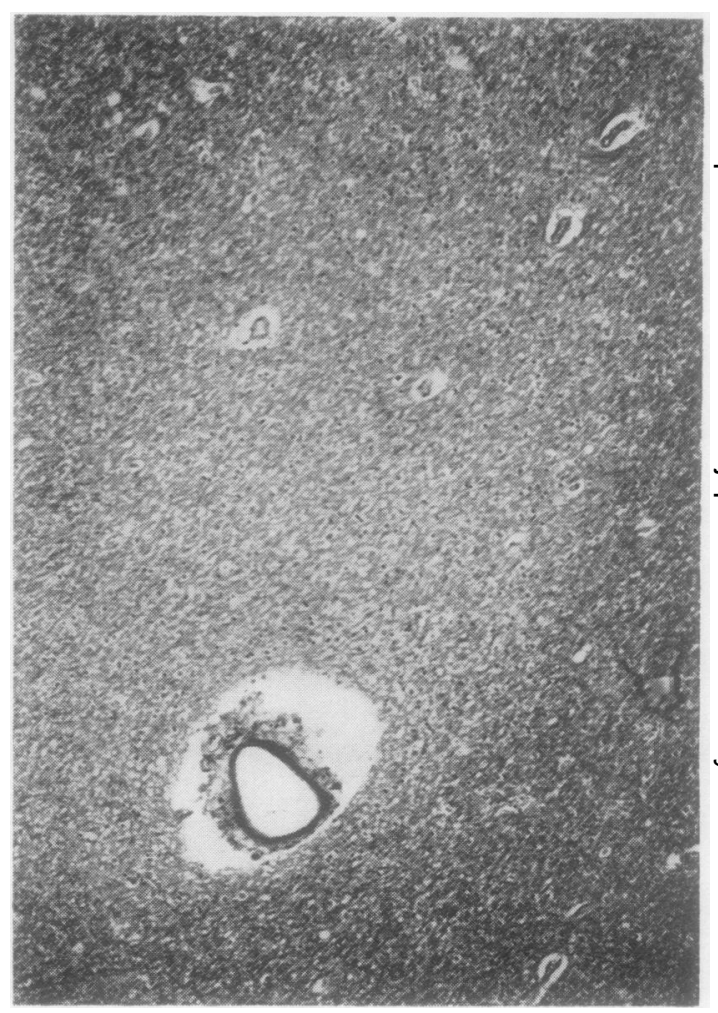

FIG. 3

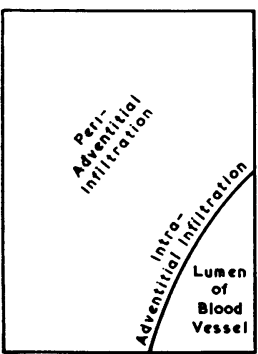

nuclei and scanty cytoplasm, also fat granule cells in various stages of formation (Fig. 2).

In general the foci were not haemorrhagic and were found in the white matter of the posterior frontal, anterior parietal and occipital lobes, also in the internal capsule, caudate and lenticular nuclei, the thalamus, mid-brain, pons, and cerebellum. The severity of the process varied throughout the brain; the mildest form, seen in the posterior part of the pons, was a narrow perivascular cuff of histiocytes with slight transudate in the adjacent neural tissue. In the more severely affected areas the perivascular demyelination had become confluent or diffuse, producing ill-defined, pale foci which showed well on Loyez and phosphotungstic-acid-haematoxylin- stained sections (Fig. 3). In the subcortical white matter of the frontal lobes, the internal capsules $N$ near the corona radiata, the thalamus, brain-stem $N$ and medulla, the demyelination was accompanied $\mathcal{N}^{\circ}$ by wide perivascular sleeves of cells (Figs. 4 and 5). In one area of the right frontal lobe, the cellular sleeves were confluent and occupied an area mea- $\stackrel{\circ}{c}$ suring $12 \times 4 \mathrm{~mm}$. (Fig. 6).

In the right dorsolateral part of the pons and the $\stackrel{+}{+}$ white matter adjacent to the dentate nucleus there was a large diffuse haemorrhagic focus of recent $\stackrel{\vec{D}}{\mathrm{D}}$ origin.

The lower medulla oblongata and the spinal cord $\stackrel{\mathbb{Q}}{\circ}$ showed a severe degenerative lesion (Figs. 8 to 17). The motor decussation of the pyramids at the 8 


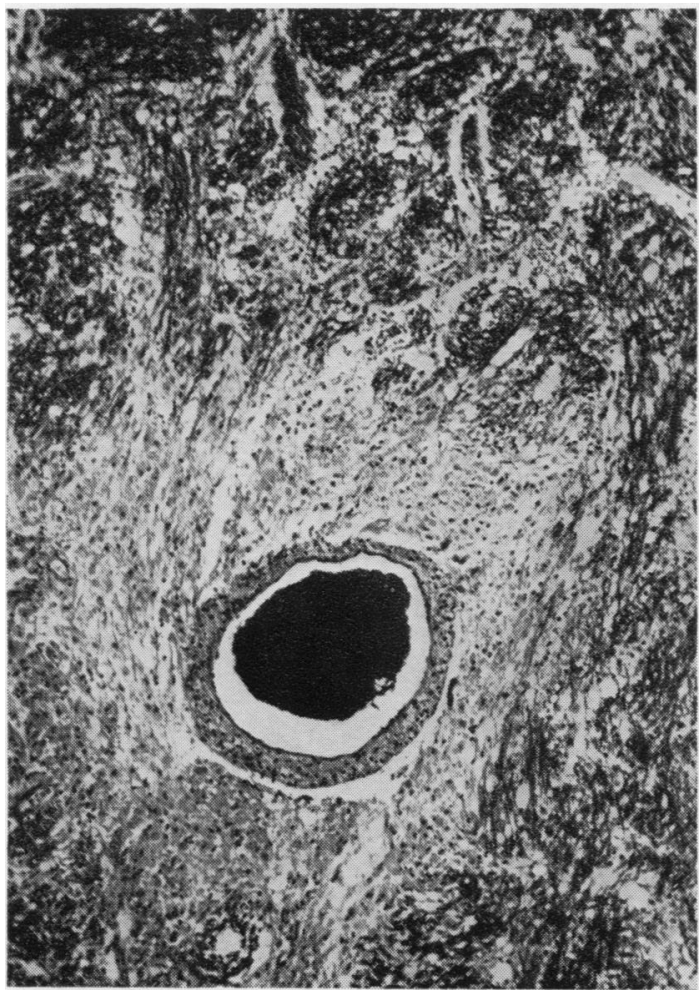

FIG. 4

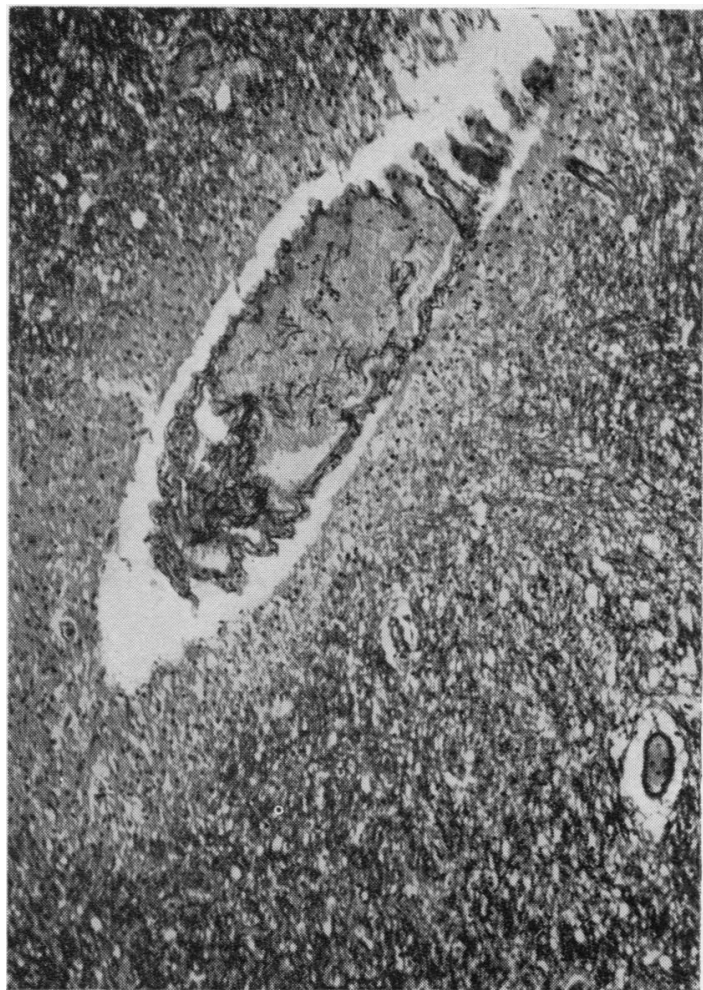

FIG. 5

acid-haematoxylin $\times 140$.)

FIG. 5. Blood vessel in the thalamus showing perivascular cellular infiltration. (Phosphotungstic acid-haematoxvlin $\times 150$.)

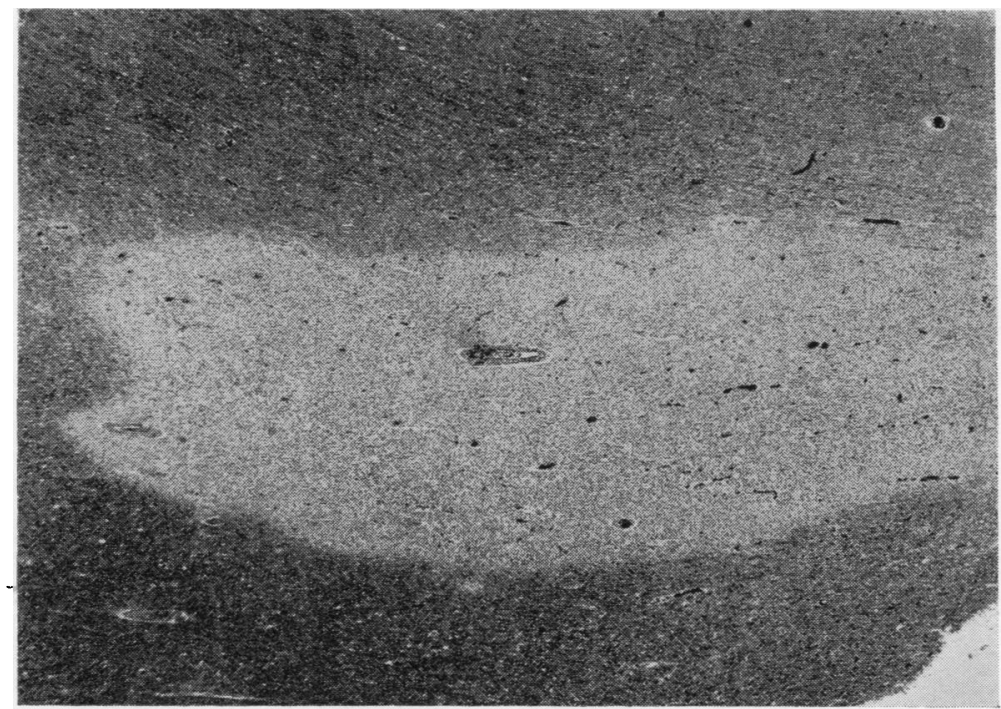

FIG. 6. A large area of confluent perivascular demyelination in subcortical white matter. (Phosphotungstic acid-haematoxylin $\times 20$.) 


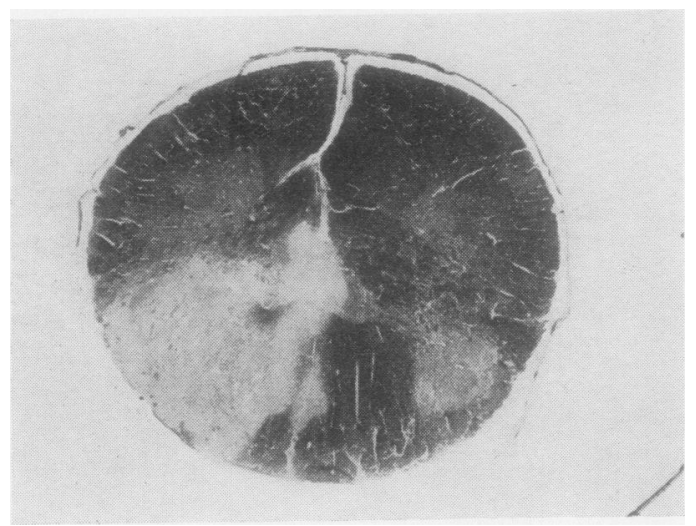

FIG. 8. Medulla oblongata at middle level of motor decussation. (Weigert-Pal $\times 15$.)

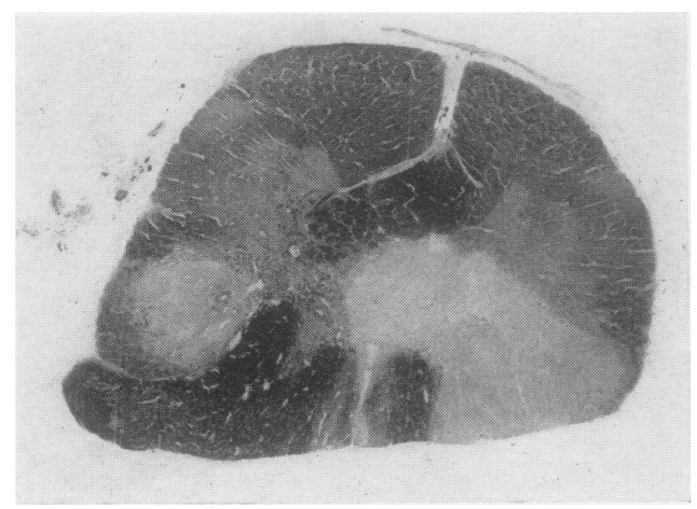

FIG. 9. Medulla at caudal level of motor decussation. (Loyez haematoxylin $\times 15$.)

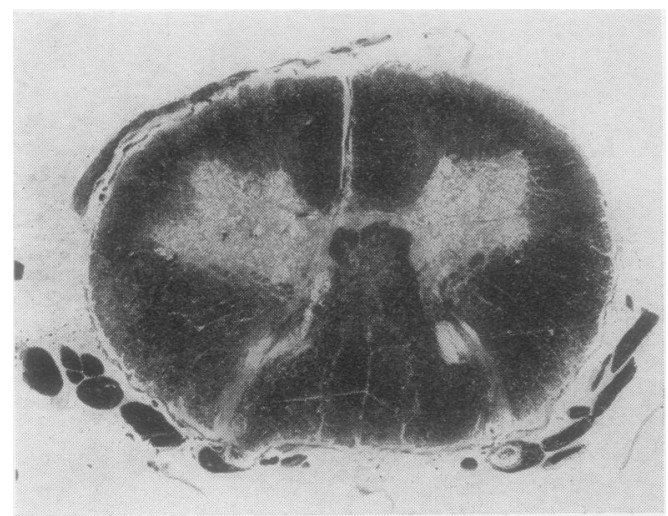

FIG. 10. Upper cervical cord showing symmetrical foci of demyelination in both posterior horns. (Weigert-Pal $\times$ 15.) caudal and middle levels of the medulla oblongata $\frac{0}{7}$ showed large areas of demyelination extending dorso-으 laterally from both sides of the central canal. The $\underset{F}{\Rightarrow}$ lesions followed a perivascular pattern and involved $\stackrel{5}{?}$ the griseum centrale, the lateral cortical and $\bar{C}$ posterior spinocerebellar tracts, the trigeminal spinal tract and nucleus, the cuneate nucleus and $\frac{\sqrt{\sigma}}{\widetilde{D}}$ funiculus, and most of the gracilis.

The upper cervical segment showed confluent ${ }_{\omega}^{\infty}$ demyelination of crossed pyramidal tracts, with less $\overrightarrow{0}$ marked degeneration in posterior white columns, $\rightarrow$ and also a small demyelinated focus in the central $\vec{\omega}$ grey matter. In the lower cervical region the grey? matter of both posterior horns was affected.

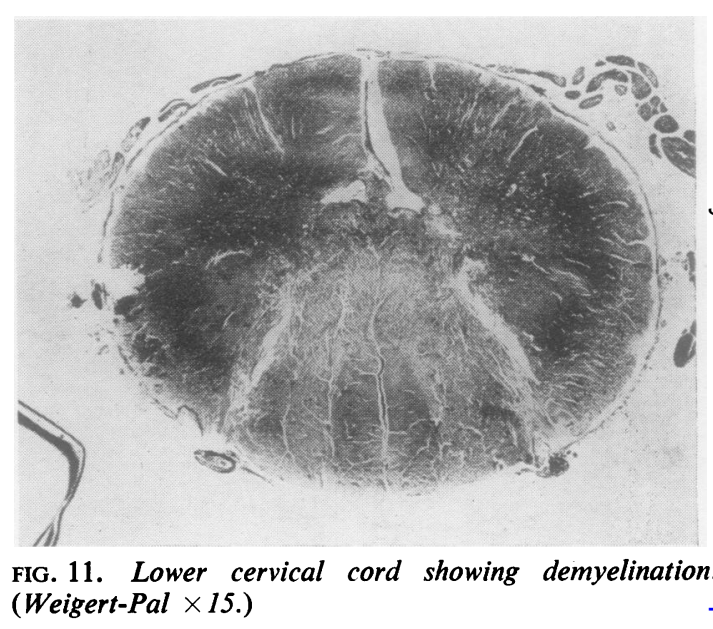

The lumbo-sacral regions contained wide con fluent areas of perivascular demyelination in most of the white matter but with sparing of the right posterior and lateral columns. Loss of myelin waš most severe in both anterior columns: there were small foci in the spinothalamic tract region and in the central grey matter of the anterior and posterio horns.

In sum, there was perivascular demyelination $\tilde{N}$ either focal or confluent, in the lower medulla oblongata and at all levels of the cord. White mattex was mainly affected, and changes were most marke ${ }^{\omega}$ in the lower cervical, dorsal, and upper lumbar regions.

Many anterior horn neurones, at all levels of the cord showed varying degrees of chromatolysis; the remainder were well preserved.

The demyelinated areas contained predominantlyo mature and immature histiocytes together with faf granule cells. Distribution was mainly perivasculae but some cells were in tissue spaces. Plasma cell, were not seen. With silver carbonate stains, the 


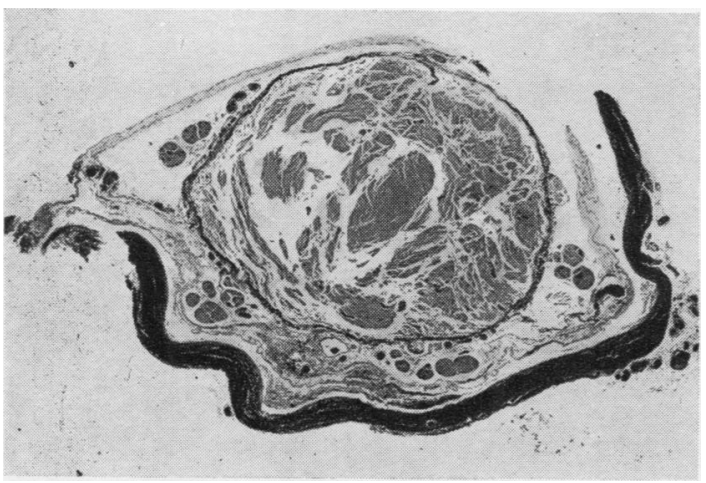

FIG. 12

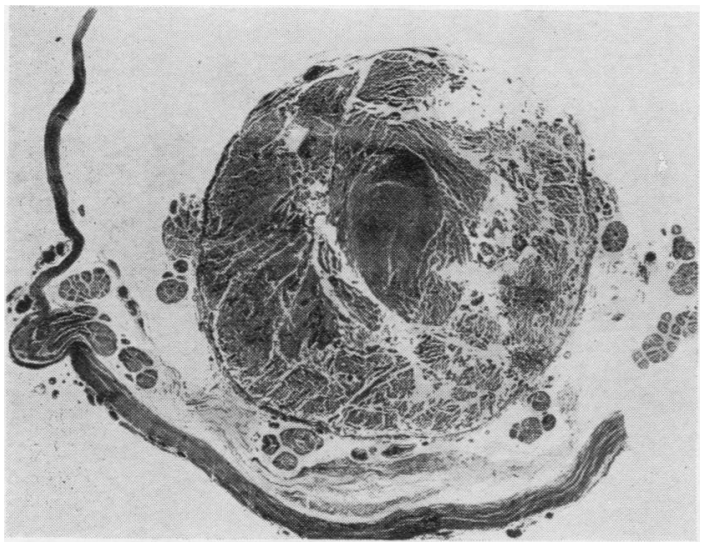

FIG. 13

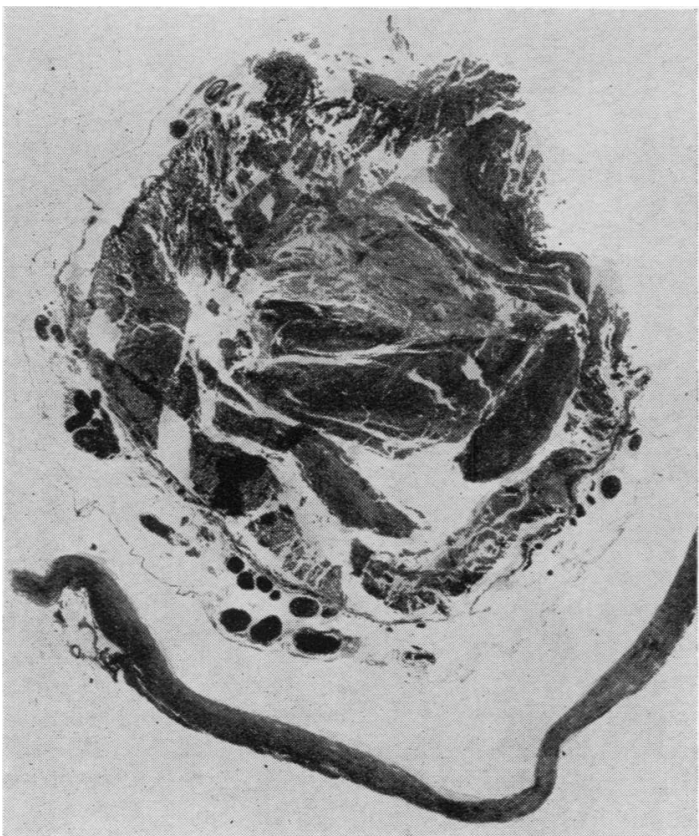

FIG. 14

FIGS. 12, 13, and 14. The upper, mid, and lower dorsal cord segments showing general loosening, disintegration, and recent acute necrosis of grey and white matter: also myelin degeneration of posterior nerve roots. (Weigert-Pal $\times$ 15.)

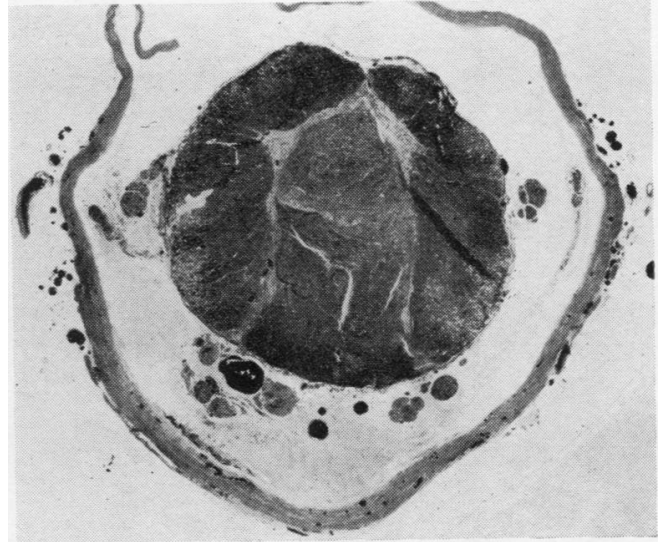

FIG. 15

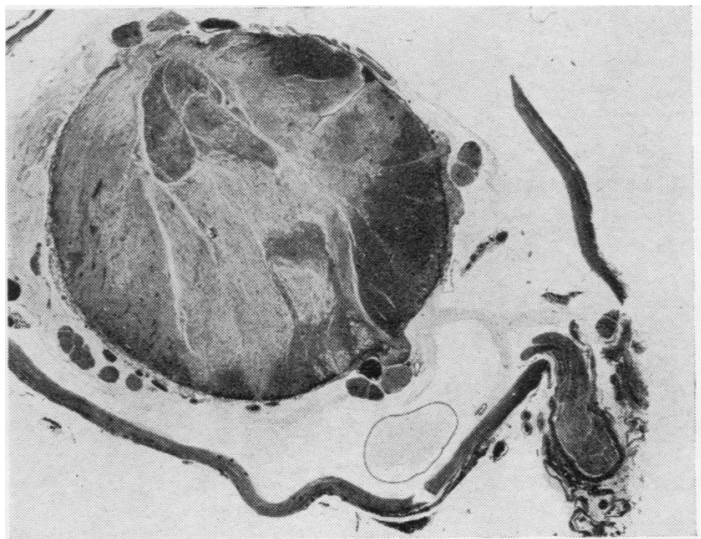

FIG. 16

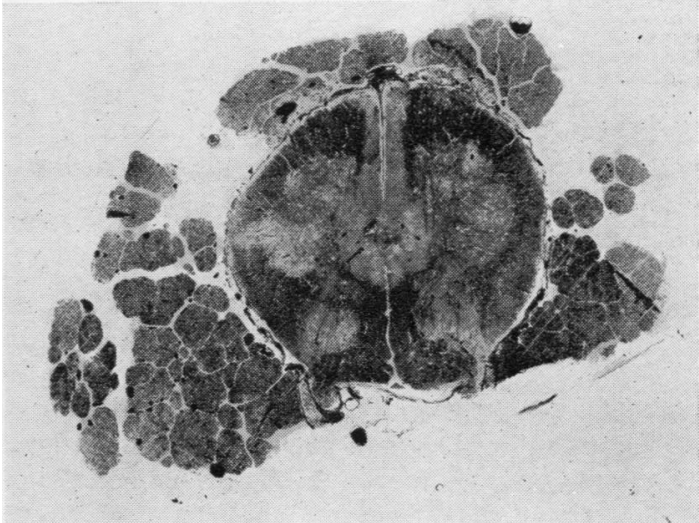

FIG. 17

FIG. 15. Another section of the dorsal cord showing less marked necrosis and demyelination. Posterior nerve roots are affected. (Weigert-Pal $\times 15$.)

FIG. 16. Mid-lumbar cord showing widespread demyelination. Note the loss of myelin in anterior and posterior nerve roots. (Weigert-Pal $\times 15$.)

FIG. 17. Lower sacral cord showing demyelination in anterior and posterior horns, central grey matter, anterior white columns, anterior and posterior nerve roots. (Weigert$\mathrm{Pal} \times 15$.) 


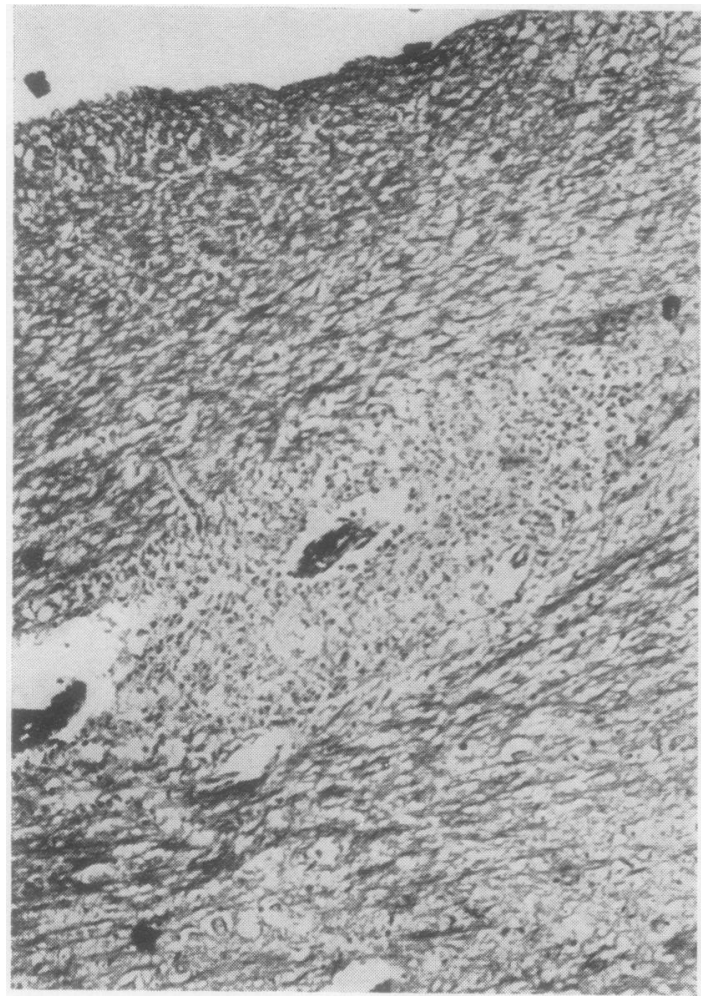

FIG. 7

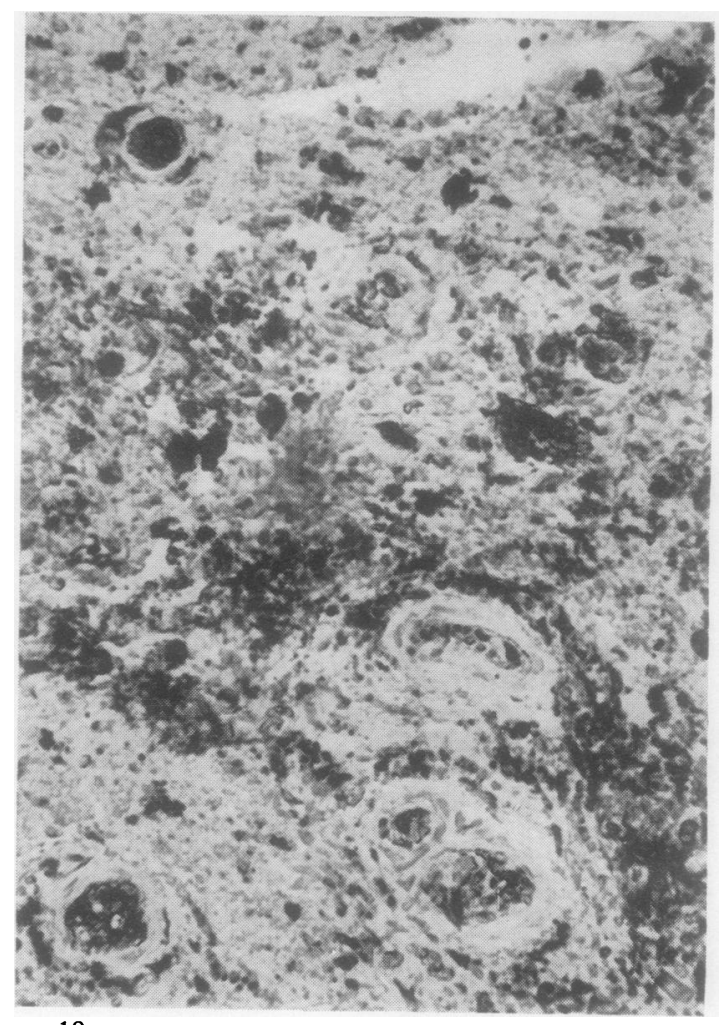

FIG. 18

FiG. 7. Blood vessel in caudal medulla surrounded by wide cellular zone. (Phosphotungstic acid-haematoxylin $\times 200$.)

FIG. 18. Recent demyelinated area in anterior white column of lumbar cord showing numerous perivascular and a few interstitially distributed histiocytes laden with lipid granules. (Sudan black $B \times 460$.)

necrosed denuded areas of the white columns in the dorsal and lumbar regions showed scanty astrocytes with swollen lobed nuclei and normal fibrils. Elsewhere in the white matter the astrocytes were better preserved.

The products of myelin catabolism stained orange red with sudan III and grey black with sudan black B; the sudanophilic material had been taken up by large histiocytes (Figs. 7 and 18) which were often arranged around blood vessels but also lay free in the necrotic tissues. Sudan black B showed marginal fat droplets in histiocytes and also demonstrated broken fragments of myelin amongst the cellular infiltration. Examination of sudan-stained sections by polarized light showed myelin balls, neutral fat droplets, anisotropic lipid droplets, and crystals which were probably cholesterol. Protein drops and decaying myelin sheaths were well shown by the periodic acid-Schiff stain.

Considering that myelin disintegration and solution is more rapid in allergic than Wallerian degen- eration (Greenfield, 1930), the stage of myelin breakdown seen in this case is compatible with the nine-day period between the onset of symptoms and death.

Silver impregnation techniques showed considerable fragmentation of some axis cylinders in the cord; elsewhere they were well preserved.

In the necrosed white matter of the dorsal and lumbar segments the perivascular demyelination and cellular infiltration was accompanied by fibrinoid necrosis of the walls of small perforating blood vessels with considerable extravasation of fibrin into surrounding neural tissues (Figs. 19, 20, and 21). These changes were also seen in a posterior nerve root. Blood vessels were intensely congested; petechial and perivascular haemorrhages were present. The leptomeninges showed little abnormality apart from slight cellular infiltrations around blood vessels in the cervical and dorsal regions.

Demyelination in the cranial nerves was marked in the third, moderately severe in the seventh and 


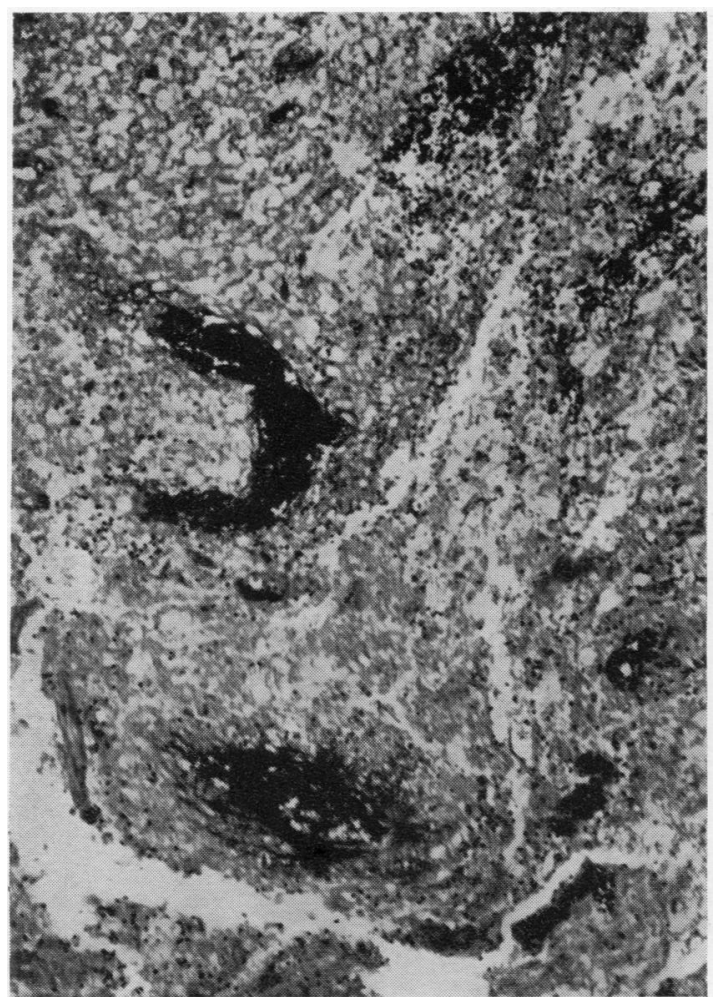

FIG. 19

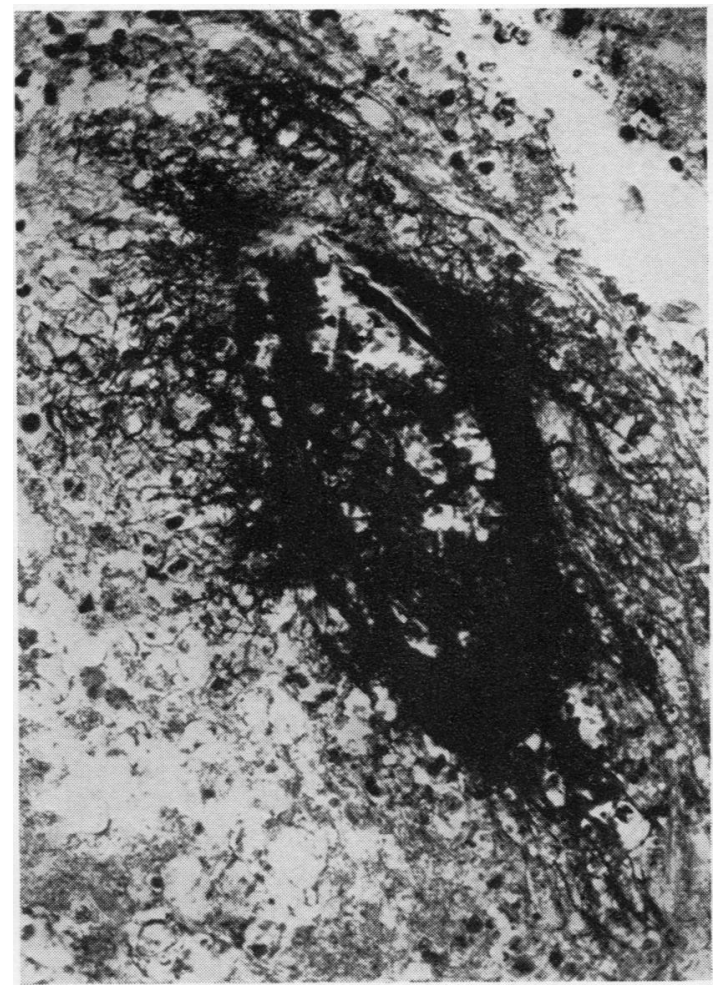

FIG. 20

FIG. 19. Necrosed area in dorsal cord s.'howing perivascular haemorrhages and infiltrations: blood vessels show fibrinoid necrosis and extravascular fibrin. (Phosphotungstic acid-haematoxylin $\times 220$.)

FIG. 20. A high-power view of one of the blood vessels in Fig. 19 demonstrating partial fibrinoid necrosis of the wall and extravasation of fibrin (stained black). (Phosphotungstic acid-haematoxylin $\times 660$.)

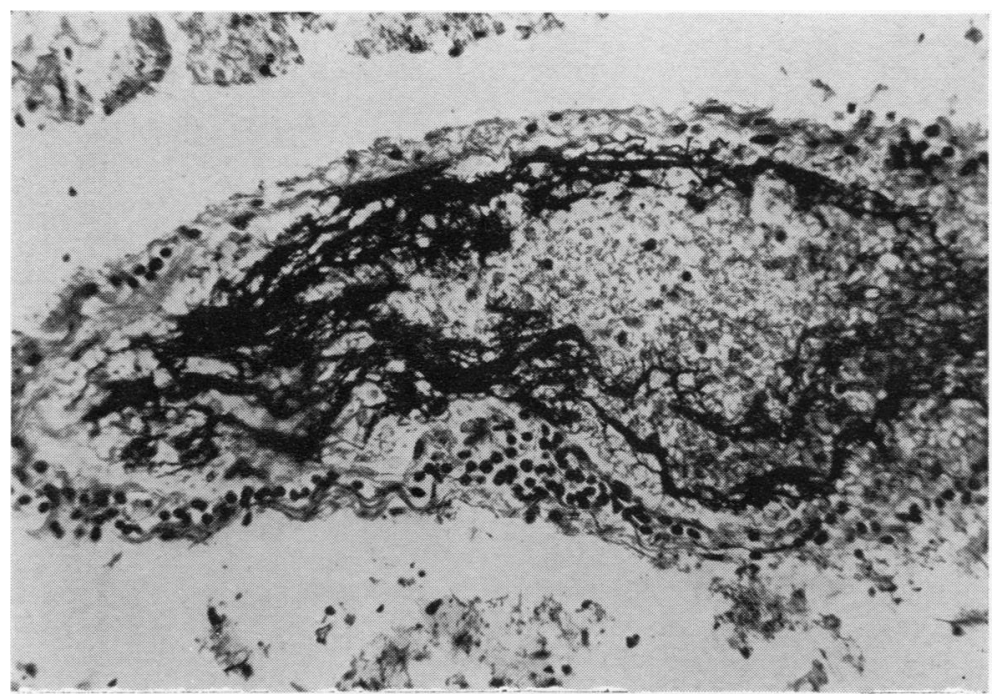

FIG. 21. Blood vessel in dorsal cord showing passage of fibrin through the necrosed wall. (Phosphotungstic acid-haematoxylin $\times 520$.) 


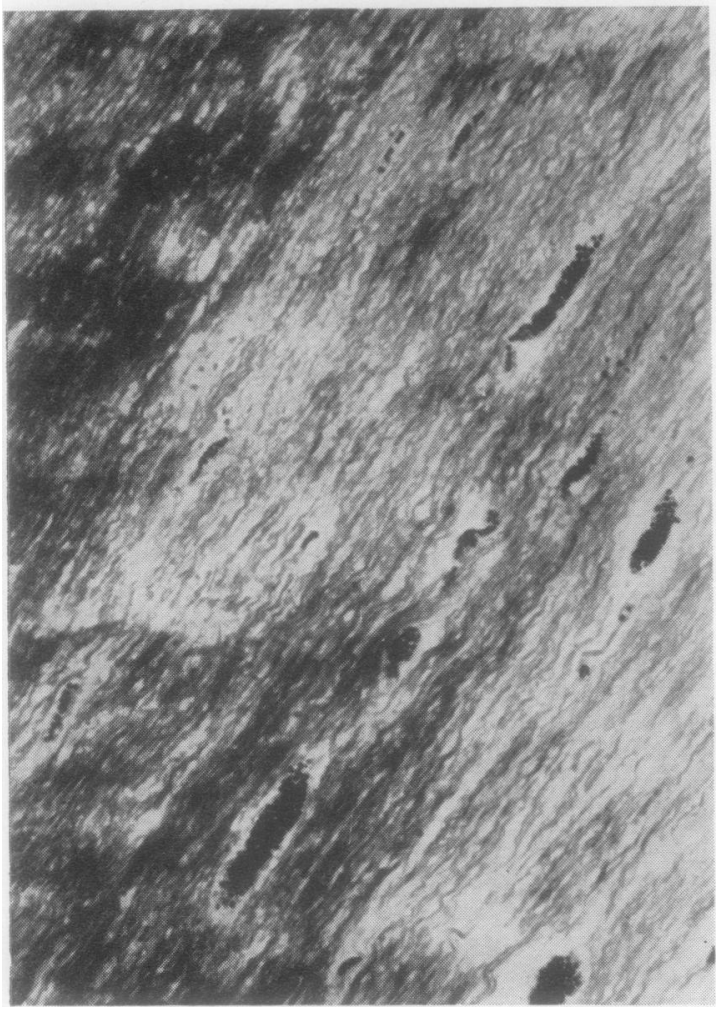

FIG. 22. Oculomotor nerve showing perivascular demyelination: cellular infiltration is absent. (Loyez haematoxylin $\times 230$.)

eighth, and less marked in the fifth and sixth. Loss of myelin was patchy and variable in the optic tracts.

The third cranial nerve showed focal and confluent perivascular demyelination without cellular infiltration (Fig. 22). There was considerable fragmentation of myelin with swollen and nodular fibres, and fat droplets and fat granule cells were present. Silver impregnation showed considerable necrosis of axis cylinders in some areas.

Posterior nerve roots at all cord levels showed patchy demyelination: the changes were severe in the dorsal and lumbo-sacral regions where myelin breakdown extended almost to the dura. Loss of myelin sheaths with the presence of myelin breakdown products was demonstrated, as was axonal decomposition. Posterior root ganglia were not affected.

A few anterior nerve roots in the lumbo-sacral region showed loss of myelin but elsewhere they appeared normal. Peripheral nerves were not examined.

\section{PATHOLOGY OF OTHER ORGANS}

The spleen was swollen and pulpy and on section $\overrightarrow{\vec{F}}$ showed intense congestion with an increased number 0 of plasma cells, some of which were atypical, $\frac{}{0}$ probably plasmablasts. The thyroid gland also showed plasma cell infiltration. Plasma cells were $\mathbb{\Phi}$ not found in sections of the lungs, heart, adrenal glands, and kidneys.

\section{DISCUSSION}

Hurst (1941) was the first to recognize acute haemorrhagic leucoencephalitis as a distinct entity, but most workers since that time have linked it with disseminated demyelinating encephalomyelitis and regard the two conditions as being kindred states $\vec{A}$ differing mainly in the violence of the assault on the central nervous system. Russell (1955) presentedthree cases which support this view; all are essentially $\subseteq$ similar to the case reported here but her third case $₹$ corresponds most closely in that there was severe $\vec{\varphi}$ damage to the brain-stem and spinal cord.

The histological features of the present case bear. a resemblance to the disseminated encephalomyelitis which can follow acute virus diseases such as smallpox and measles, can result from vaccination against smallpox and rabies, and has also been seen $\frac{\varnothing}{\circ}$ as a sequel to an 'influenza-like' illness (Greenfield, $\stackrel{2}{\overrightarrow{7}}$ 1930). In the post-vaccinal cases reported by Turnbull and McIntosh (1926) the characteristic zones of demyelination and cellular infiltration were accompanied by petechial haemorrhages (Russell, 1943). One of the two 'post-influenza' cases reported by용 Greenfield (1930) showed petechial haemorrhage, $\overline{-}$ but extravasation of fibrin, as seen in the case described here, was not present in either.

The confluent demyelination of the medulla oblongata and the massive necrosis of the white ? matter of the cord which are prominent features of $>$ this case were not found in any of the cases reported by Russell (1955), nor in previous cases reviewed by $N$ her in the same paper: since that time there is no record of a similar case.

In the numerous sections examined it would $N_{\omega}^{N}$ appear that the lesions throughout the brain, medulla, and spinal cord were about the same age.co These findings make the diagnosis of multiple, $\overparen{D}$ diffuse, or disseminated sclerosis untenable, as does? the presence of fibrinoid necrosis in blood vessels.

Acute necrotic encephalopathy, as described by

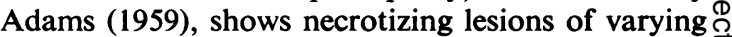
ages and is thus dissimilar to the case reported here. $\stackrel{\mathbb{D}}{\circ}$ The clinical and pathological features of acute $O$ necrotic myelopathy (Hoffman, 1955) are essentially different from our findings. The vascular changes $\frac{0}{0}$ 
present in this case also rule out neuromyelitis optica although there is some similarity in the patchy demyelination of the optic tracts.

The findings of haemorrhage, perivascular demyelination, and cellular infiltration, together with fibrinoid necrosis of blood vessel walls and extravasation of fibrin, adequately fit the diagnostic picture of acute disseminated encephalomyelitis and haemorrhagic leucoencephalitis. The features of this case appear to support the tenet that these two conditions are facets of the same pathological entity.

The many parallels between experimental encephalomyelitis and some aspects of human demyelinating diseases warrant the view that the latter are essentially allergic disorders. Wolf (1952), Russell (1955), and Adams (1959), and many other European and American workers, are satisfied that experimental allergic encephalitis represents a satisfactory laboratory model for acute disseminated encephalomyelitis and haemorrhagic leucoencephalitis in man.

The findings in this case suggest that the process was a neuroallergic reaction of unusual severity. The acutely inflamed spleen which, together with the thyroid gland, contained an excess of plasma cells, support the allergic theory (Campbell and Good, 1950).

The only possible antigenic substance in this case was a single subcutaneous injection of antitetanus serum given 15 days before the onset of symptoms. There is no evidence in the available literature that penicillin can produce such phenomena.

The patient had never before received horse serum. The only prophylactic injections given were of poliomyelitis vaccine: she had not been vaccinated.

There are many reported cases of neurological sequelae following injections of serum. Baker (1942) described 12 cases of tetanus, some treated with antitoxin, which showed perivascular demyelination in the brain: Putman, McKenna, and Morrison (1931) produced similar results in dogs by repeated injections of tetanus antitoxin. Csermely (1950) described one case of haemorrhagic and nonhaemorrhagic lesions of acute disseminated demyelinating encephalomyelitis following the use of antitetanus serum: the reactions were less severe than in this case but were essentially similar. Miller and Stanton (1954), in a review of the literature on neurological sequelae of prophylactic innoculation, include three cases in which antitetanus serum was used.

A case of acute necrotizing haemorrhagic encephalopathy following the administration of tetanus antitoxin is described by Toogood (1960) but histological findings are not given. Williams and Chafee (1961) describe demyelinating encephalomyelitis in a case of tetanus, treated with antitoxin, in a boy aged 13 who showed urticaria and angioedema on the eighth and eleventh days after the injections. He became comatose and died on the thirteenth day.

The case reported here, along with the others mentioned, appear to represent manifestations of the delayed type of allergic response (Waksman, 1959).

Our thanks are due to H.M. Coroner for permission to publish this case. We are most indebted to Dr. L. Rubinstein for reviewing the histology, making the diagnosis, and referring us to the literature; to Dr. $\mathrm{H}$. Urich for many helpful suggestions and for reading the manuscript; to Mr. R. A. Daws and Mr. R. S. Garden for access to clinical history; to Messrs. J. H. Wilkinson and $\mathbf{J}$. Urquhart for preparing the large amount of histological material, and Mr. John King of the London Hospital for photomicrographs.

\section{REFERENCES}

Adams, R. D. (1959). In "Allergic" Encephalomyelitis, ed. M. W. Kies and E. C. Alvord, pp. 187-207. Thomas, Springfield, Ill.

Baker, A. B. (1942). J. Neuropath. exp. Neurol., 1, 394.

Campbell, B., and Good, R. A. (1950). Arch. Neurol. Psychiat. (Chicago), 63, 298.

Csermely, H. (1950). Ibid., 64, 676.

Greenfield, J. G. (1930). J. Path. Bact., 33, 453.

Hoffman, H. L. (1955). Brain, 78, 377.

Hurst, E. W. (1941). Med. J. Aust., 2, 1.

Miller, H. C., and Stanton, J. B. (1954). Quart. J. Med., 23, 1.

Putman, T. J., McKenna, J. B., and Morrison, L. R. (1931). J. Amer. med. Ass., 97, 1591.

Russell, D. S. (1943). Proc. roy. Soc. Med., 36, 321.

(1955). Brain. 78, 369.

Toogood, J. H. (1960). Canad. med. Ass. J., 82, 907.

Turnbull, H. M., and McIntosh, J. (1926). Brit. J. exp. Path., 7, 181.

Waksman, B. H. (1959). In "Allergic" Encephalomyelitis, ed. M. W. Kies and E. C. Alvord. Thomas, Springfield, Illinois.

Williams, H. W., and Chafee, F. H. (1961). New Engl. J. Med., 264, 489.

Wolf, A. (1952). Proc. First int. Congr. Neuropath., Rome, 1952, vol. $1,121$. 